\title{
MULTILANGUAGE SOURCE CODE GENERATOR FOR DATABASE ORIENTED APPLICATION USING JSP
}

\author{
J. Jagadeesan ${ }^{1}$, G.M. Kadhar Nawaz ${ }^{2}$ \\ ${ }^{1}$ Assistant Professor, Department of Computer Science, Kanchi Mamunivar Centre for P.G. Studies, (Autonomous), \\ Govt. of Puducherry, Lawspet, Puducherry-605 008, India \\ ${ }^{2}$ Director \& Professor, Department of MCA, Sona College of Technology, Salem - 636 005, Tamilnadu, India
}

\begin{abstract}
The goal of automated software engineering is to increase by order of magnitude both the quality and productivity factor. To date most successful approach is producing a special-purpose code generator, such as screen generator, report generator, swing builder, Meta programming etc., these software tools is produce source code for specific language. This study provides mechanism to make a generalized system of Multi-Language Source Code Generation (MLSCG). It takes database structure of metadata as input and produce source code in Java, Java script, JSP, PHP and ASP. We utilize the target output code for both web and pc-based application.
\end{abstract}

Keywords: automatic programming, source code generation, JSP, PHP, ASP and database.

\section{INTRODUCTION}

The current computer software industry, there have been interested to attempts to create the software for autoprogramming. The end user oriented general purpose, fully automatic programming is possible. For this reality we need to fulfill this approach such as Bottom up, Narrow domain and Assistant. The act of generating source code based on an ontological model, However the template and is accomplished with a programming tool[1].

The source code generator is defined as a multi level tree structure where a higher-level generator is given by the superposition of lower-level generators. Several generators can share the same application specification. It giving different outputs of source code. Moreover, some polymorphic features are provides similar to late binding of virtual methods in Object-Oriented Programming. A procedure can propose late binding of code Templates during the process of generation, which makes it easier to add new features to program Specification. The end-user point of view we need to complete their requirements [2].

\section{RELATED WORKS}

The Table 1 shows some of the existing code generator (CG). The serial number 1 to 6 code generation built in Integrated Development Environment (IDE) itself, remaining are the separate software format. Most of the software gives frame work of the project such as templates. In which FoxPro is the database management system, after selecting database it allow us to build screen, report and query.[3]

In Visual Basic 6.0 provides the facility to generate application wizard. This is step by step selection of database, field and build form screen. The generated screen consist of insert, delete modify and select data from table. The third software in the Table 1 is Front Page Express and Visual Inter Developer Studio; both of the software are Micro Soft products. It is having facility to design the form and it generates Hyper Text Markup Language (HTML) code for that design. Dreamweaver is the macromedia product. It also generates HTML code [4]. Similar source code generation products are generates static source code for various programming languages.

\subsection{The Meta-Base}

The meta-base comprises an extended data model. The programming language description and some of additional information to support the generation process [5]. This type of meta-based generators produces database schema, application programs and user interfaces. Meta data can be generated and automatically stored into the meta-base. For instance, relationships between entities are generated based on keys attributes, and forms are generated based on the data model definitions and predefined form generation parameters [6].

\subsection{Regular Expression to CG}

The semantics refer to the meaning of modeling elements (symbols) and how they interpreted. We have to validate the expression, then extract the symbol from regular expression and create the symbol table. Based on symbol table the code generator writes definition and input statements. CG can produces generates the source code for the given assignment statement [6]. The Regular Expression (RE) to source code has been implemented in FoxPro. The screen generation module in FoxPro consists of RE based CG. The output field variable in FoxPro allow us the expression building for RE.[7] 
Table 1: Code Generation Software

\begin{tabular}{|c|c|c|}
\hline $\begin{array}{l}\text { Sl. } \\
\text { No. }\end{array}$ & $\begin{array}{l}\text { Package/ } \\
\text { Language } \\
\text { Name }\end{array}$ & Type of Code Generation \\
\hline 1. & FoxPro 2.6 & $\begin{array}{l}\text { Screen Generator } \\
\text { Report Generator }\end{array}$ \\
\hline 2. & Visual Basic 6.0 & Database Code Generator \\
\hline 3. & \begin{tabular}{|lr} 
Front & Page \\
Express, & Visual \\
InterDev. & \\
\end{tabular} & Screen Generator \\
\hline 4. & Dreamweaver & $\begin{array}{l}\text { Screen Generator } \\
\text { Database Code Generator }\end{array}$ \\
\hline 5. & Netbean IDE & $\begin{array}{l}\text { Swing Builder, } \\
\text { A standard project uses an IDE- } \\
\text { generated build script to build, } \\
\text { run, and debug your project. }\end{array}$ \\
\hline 6. & Eclipse SDK & $\begin{array}{l}\text { Model Fragment, } \\
\text { Customized declaratively using } \\
\text { Cascading Style Sheets (CSS), }\end{array}$ \\
\hline 7. & $\begin{array}{l}\text { Interface } \\
\text { Builder }\end{array}$ & $\begin{array}{l}\text { Formerly Project Builder for } \\
\text { Apple's, and iPhone. }\end{array}$ \\
\hline 8. & $\begin{array}{l}\text { Code Smith } \\
\text { Generator }\end{array}$ & $\begin{array}{l}\text { Database schema to C\# or } \\
\text { VB.NET source code. }\end{array}$ \\
\hline 9. & \begin{tabular}{|lr} 
Apeel & PHP \\
Code & Generator \\
Pro &
\end{tabular} & $\begin{array}{l}\text { PHP, CSS, Apeel Open Source } \\
\text { Framework. }\end{array}$ \\
\hline 10. & JCodeBox & $\begin{array}{l}\text { It helps maximize the productivity } \\
\text { of Java IDE. }\end{array}$ \\
\hline
\end{tabular}

\subsection{Screen and Report Generators}

The screen and report generators can receive screen design objects stored into a composed section. The screen input consists of Text, Input variable, expression, output variable and memory variable. Simultaneously it should store the sequence of the screen object and create a set of tasks. Each task consists of set of attributes. In case of report generator has three portions such as Heading, Report body and footer. These report elements also converted into set of task for CG [4].

\subsection{Database to Code Builder}

The database to code builder is used to query handling and report generation. In this type of builder has presented in macromedia dream weaver software. This package can produced code for dynamic record set handling for ASP.NET and PHP language.

\subsection{Net Beans IDE}

This IDE allows the user to create project for Java, JEE, PHP and $\mathrm{C}++$. It generates templates and related interface files for new projects. While type java code it compiles simultaneously, make under line for error tokens, display auto text for package reference, Refactor the entire project at the time of renaming, etc., Swing form building is one of the features in this IDE $[4,8]$.

\subsection{Algorithmic Code Generation (ACG)}

Algorithmic Code Generation software is having information about corresponding programming-languagesyntax for algorithmic statement. By using this information ACG generates the code shortly in any language [9].

\subsection{Limitations in Present CG System}

- $\quad$ From above code generation system, it produces static code.

- $\quad$ Some system allows us to generate code by wizard, after extension of programming in the generated code; if we need any modification, we have to start the work from beginning stage of the wizard.

- The programmer is feels difficult to maintain machine generated variables.

- The user should read all the bulk amount of generated source code for extend the code.

\section{PROPOSED WORK}

\subsection{Overview}

The first module of the Multi-Language Source Code Generator (MLSCG) receives the input(database structure) of user requirements and generate source code by using of Target Language Description (TLD) information. The Figure-1 shows the goal diagram of proposed work that has to be achieved to reach the main goals. In which TLD is consists of program templates, database connectivity structure, query code and program statement syntax information. MLSGC is responsible to fetch the suitable code from TLD. Each module is decomposed until the lower level goal can be achieved by accomplishing simple action.

The generated code have the added information about Comment of Author, Company, Address, City, Date of generation, theme selection, target folder, debugging information, etc., Some developer needs report with/without table. The MLSGC is providing feature of flexible report format.

\subsection{HTML JavaScript}

The MLSGC gives Java and HTML with JavaScript code as a Personal Computer(PC) based application type. Some user needs the software within PC. The application in HTML with JS format is the very simple coding techniques. There is no need of special compiler to execute these coding. The browser is sufficient for execute these coding. This type of source code is useful to un-securable database application. This system is securable for data. The code is open source model. 


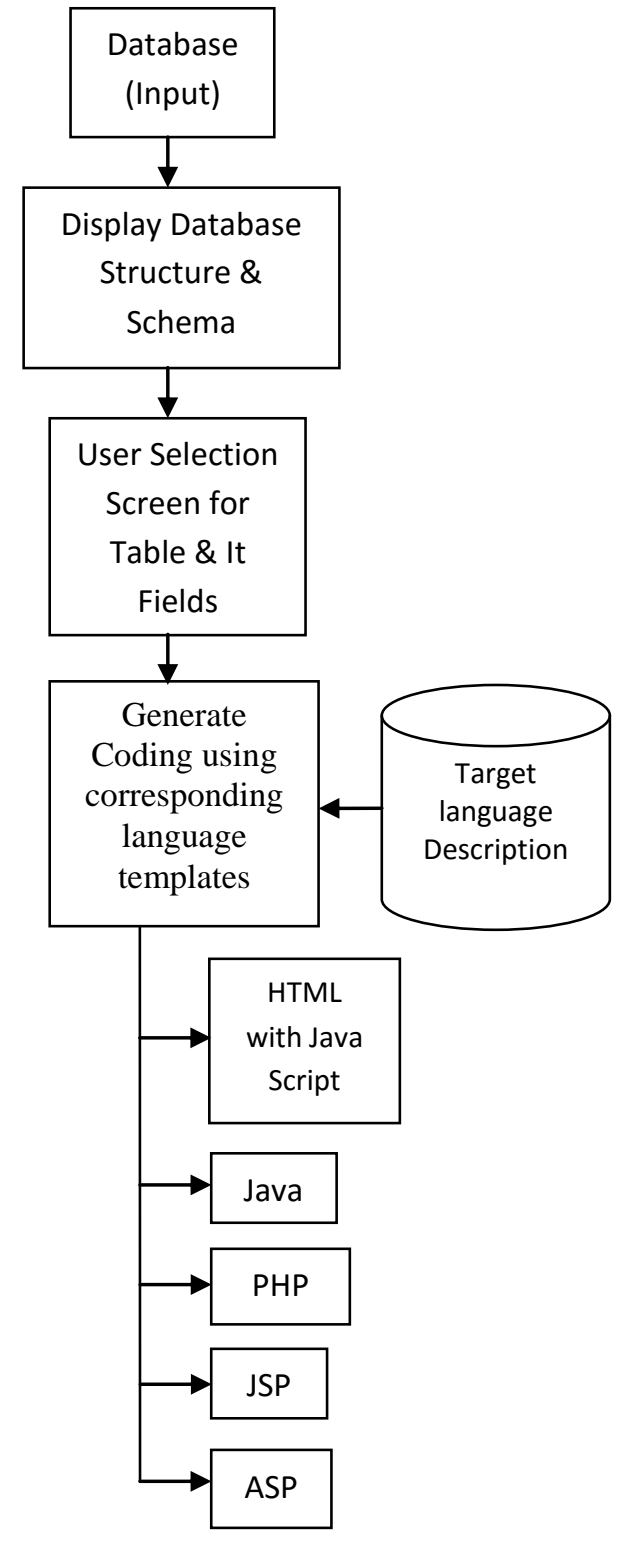

Fig 1 Overview of proposed work

\subsection{The Java-Swing}

The Java-Swing is one of the target language. After the achieved the code we can easily pluggable. However it is a Lightweight components, It do not depend on native peers to render themselves, simplified graphics screen, similar behaviour across all platforms, portable look and feel, similarly only a few top level containers not lightweight. It has the new text components tooltips, textual popup to give additional help and arbitrary keyboard event binding with debugging support.

\subsection{The JSP Code within JSP}

The Java Server Pages (JSP) is most powerful language. All JSP code will go for run after conversion of Servlet. It has the all features of Java and added features of web server. In the proposed work, at time of developing main modules of JSP code generator the syntax may be conflict. However the source and target language is same. The following example code shows this problem of double quote. We have to give systemic treatment of double quote as escape sequence. It is a complex task to code with in code.

\section{Need Output Code}

Out.println("Process ID : “ + pid);

Out.println("out.println("Process ID: " + pid); ");

Out.println(“"out.println(l“Process ID: ‘“+ pid); “);

\subsection{The PHP output code}

PHP manages freeing all types of database resources. Users does not required to free file handle resource, database resources, memory, etc, unless programmer need to free resource during script execution. We control which portability modes are enabled by using the portability configuration option. Configuration options are set via connect() and setOption(). The Table 2 shows target language description generated by the proposed work. The code type of PHP is web based application, the entry screen and reports are HTML with CSS.

Table 2: Target Language output description.

\begin{tabular}{|c|l|l|l|l|l|}
\hline \multirow{2}{*}{$\begin{array}{c}\text { Sl } \\
\text { N }\end{array}$} & \multirow{2}{*}{$\begin{array}{c}\text { Langua } \\
\text { o. }\end{array}$} & \multicolumn{4}{|c|}{ Target Language Description } \\
\cline { 3 - 6 } & $\begin{array}{c}\text { Code } \\
\text { Type }\end{array}$ & $\begin{array}{c}\text { Entry } \\
\text { Screen }\end{array}$ & $\begin{array}{c}\text { Database } \\
\text { Connection }\end{array}$ & Report \\
\hline 1. & Java & $\begin{array}{l}\text { PC based } \\
\text { application }\end{array}$ & Swing & JDBC-Java & Swing \\
\hline 2. & $\begin{array}{l}\text { HTML } \\
\text { with JS }\end{array}$ & $\begin{array}{l}\text { PC based } \\
\text { application }\end{array}$ & $\begin{array}{l}\text { HTML } \\
\text { with } \\
\text { CSS }\end{array}$ & Java Script & $\begin{array}{l}\text { HTML } \\
\text { with JS }\end{array}$ \\
\hline 3. & JSP & $\begin{array}{l}\text { Web based } \\
\text { application }\end{array}$ & $\begin{array}{l}\text { HTML } \\
\text { with } \\
\text { CSS }\end{array}$ & JDBC-JSP & $\begin{array}{l}\text { HTML } \\
\text { with } \\
\text { CSS }\end{array}$ \\
\hline 4. & PHP & $\begin{array}{l}\text { Web based } \\
\text { application }\end{array}$ & $\begin{array}{l}\text { HTML } \\
\text { with } \\
\text { CSS }\end{array}$ & PHP & $\begin{array}{l}\text { HTML } \\
\text { with } \\
\text { CSS }\end{array}$ \\
\hline 5. & ASP & $\begin{array}{l}\text { Web based } \\
\text { application }\end{array}$ & $\begin{array}{l}\text { HTML } \\
\text { with } \\
\text { CSS }\end{array}$ & ASP & $\begin{array}{l}\text { HTML } \\
\text { with } \\
\text { CSS }\end{array}$ \\
\hline
\end{tabular}

\subsection{MLSCG-Phases}

In the final phase of MLSCG is having the following algorithm.

\section{Phase I}

Read Database metadata and store it into session variable.

Raise Entry screen for table and field selection Store selected table \& field into session Read primary, foreign key of table and store it into session. 
Raise Entry screen for language selection

Store selected languages into session

\section{Phase II}

Read session data into memory variable

For $\mathrm{i}=1$ to no. of fields

Insert field name

Create input entry code

Create database connectivity code

Generate corresponding(Select/Insert/Delete/Update) SQL statement

Create Loop statement until end of file

For $\mathrm{i}=1$ to no. of fields

Display field

Close data base

\subsection{Importance of Data type}

MLSCG system is concentrated about suitable data type corresponding to the table structure and programming languages. It is the second advantage of MLSCG system. It gives skeleton of the proposed work. The Table 3 shows the matching of database data type with language data type. This system covered most widely using data types. Any new data-type presents in the input the system raise the comments for particular code. The user may alter the code to suitable data type. Another way before the database structure input alter the data type in table structure.

\subsection{Report Generation Phase}

In this phase generate the query using primary key of table description. The following algorithm is used for report generation phase.

Table 3: Matching of Database data-types with Language data-types.

\begin{tabular}{|l|l|l|l|l|l|}
\hline $\begin{array}{l}\text { My } \\
\text { SQL }\end{array}$ & Access & Java & $\begin{array}{l}\text { Java } \\
\text { Script }\end{array}$ & PHP & ASP \\
\hline Int & Single & short & short & free & Short \\
\hline Bigint & Integer & Int & Int & free & Integer \\
\hline $\begin{array}{l}\text { Long } \\
\text { Integer }\end{array}$ & $\begin{array}{l}\text { Long } \\
\text { Integer }\end{array}$ & Long & Long & free & Long \\
\hline Double & Double & Double & Double & free & Double \\
\hline Boolean & Yes/No & Boolean & Boolean & free & Boolean \\
\hline $\begin{array}{l}\text { Date- } \\
\text { time }\end{array}$ & $\begin{array}{l}\text { Date- } \\
\text { time }\end{array}$ & Date & Date & free & Date \\
\hline Char & Text & Char & Char & free & Char \\
\hline Varchar & Text & String & String & free & String \\
\hline Text & Text & String & String & free & String \\
\hline $\begin{array}{l}\text { Long } \\
\text { Text }\end{array}$ & Memo & String & String & free & string \\
\hline
\end{tabular}

Create HTML elements

Insert CSS related links

Display selected fields for Heading

Open Database

Generate Select query with primary key for where clause.

Insert while loop code until eof()

Display selected fields

Close database

\subsection{Features}

* Single click source code generator

* Produce 3 Dimensional codes, such as we are giving code for client, web tire and server.

* Generated codes are in the form of text file. They user may modify their coding according their convenience.

* It consist of simple and flexible Report Generator

* This will helpful for software developers.

\section{CONCLUSION}

The automatic code generator using JSP allows the programmer to create maintainable and reusable code, eventually improving their productivity. The proposed work is done major advantage for developer. We achieve this logic building in some extend by our main module. Even though MLSCG the significant benefits and the profound logic search code impact of this work in the software development field. Good representation of user problem description eliminate irrelevant source code output, capture relevant distinctions, and express knowledge at the most general level possible.

In future, the proposed of MLSCG design work will be implement by using Java and JEE languages. Java has the significant features such as Object Oriented Programming. These features will be suitable for future source code generator design.

\section{REFERENCES}

[1]. Danijel, "Source Code Generator Based on Dynamic Frames", JIOS, Vol. 35, No.1(2011)

[2]. Charles Rich and Richard C. Waters, "Automatic Programming: Myths and Prospects", 0018- 9162/88/08000040\$01.00 IEEE, 1988.

[3]. Daniel L. Silver, "Machine Lifelong Learning: Challenges and Benefits for Artificial General Intelligence" J. Schmidhuber, K.R. Th'orisson, and M. Looks (Eds.): AGI 2011, LNAI 6830, pp. 370-375, 2011.

[4]. Code Generation Tools, June 2013, http://en.wikipedia.org// Comparison_of_code_ generation_tools

[5]. Yuri B Danilchenko, "Automatic Code Generation Using Artificial Intelligence."

[6]. Krešimir Fertalj, Damir Kalpić, Vedran Mornar, "Source Code Generator Based on a Proprietary Specification Language", 0-7695-1435-9/02 \$17.00 (c), IEEE, 2002

[7]. Muhammad Usman, and Aamer Nadeem, "Automatic Generation of Java Code From UML Diagrams Using UJECTOR", International Journal of Software Engineering and its Applications, Vol.3, No.2, April 2009.

[8]. Rashmi Sinha and Ashish Dewangan, "An Approach for Code Generator- ENUM," ISSN (Print) : 2319 -2526, Volume-2, Issue-5, 2013.

[9]. Amit Barve and Brijendra Kumar Joshi, "Automatic C Code Generation for Parallel Compilation", Page 1, ISSN (Print) : 2319 - 2526, Volume-2, Issue-4, 2013. 